\title{
HORMIGAS (HYMENOPTERA: FORMICIDAE) ASOCIADAS A PALMA CAMEDOR (CHAMEDOREA RADICALIS MART.) EN EL BOSQUE TROPICAL, GÓMEZ FARÍAS, TAMAULIPAS, MÉXICO
}

\section{Manuel LARA-Villalón, ${ }^{1}$ Madai ROSAS-MEJíA, ${ }^{1}$ Patricia ROJAS-FERNÁNDEZ ${ }^{2}$ y Pedro REYES-CASTILLO ${ }^{2}$}

\author{
${ }^{1}$ Instituto de Ecología Aplicada. División del Golfo No. 356, 87019, Ciudad Victoria, Tamaulipas, México. \\ <mlarav@uat.edu.mx> \\ ${ }^{2}$ Instituto de Ecología A.C., Red Biodiversidad y Sistemática, Km. 2.5 Antigua carretera a Coatepec 351, 91070, \\ Xalapa, Veracruz, México. \\ Recibido: 20/12/2014; aceptado: 10/02/2015
}

Lara-Villalón, M., Rosas-Mejía, M., Rojas-Fernández, P. \& Reyes-Castillo, P. 2015. Hormigas (Hymenoptera: Formicidae) asociadas a palma camedor (Chamedorea radicalis Mart.) en el bosque tropical, Gómez Farías, Tamaulipas, México. Acta Zoológica Mexicana (n. s.), 31(2): 270-274.

RESUMEN. La diversidad de hormigas asociadas al cultivo de la palmilla o palma "camedor" Chamaedorea radicalis Mart., bajo un sistema agroforestal, ha sido estudiado en el bosque tropical de la Reserva de la Biosfera El Cielo (RBEC). Se colectaron en las frondas mediante red entomológica de golpeo, las siguientes especies en dos épocas estacionales: Odontomachus laticeps, Crematogaster cerasi, Camponotus atriceps, Temnothorax texanus, Tapinoma sp., y Pheidole arctos en época lluviosa. Wasmannia auropuntata, Camponotus atriceps, Monomorium floricola, Pheidole arctos y Solenopsis geminata en época seca. Se comparan las especies reportadas por otros autores en la RBEC. En conjunto los géneros encontrados son colonizadores de agroecosistemas, con hábitos forrajeadores, cazadoras, recolectoras de néctar, semillas y restos de animales muertos. El conocimiento de los organismos asociados a la palma camedor y su ambiente, permitirá establecer un manejo integrado de plagas para la sustentabilidad de este importante biorrecurso.

Palabras clave: diversidad, registro de especies, plagas.

\section{INTRODUCCIÓN}

Durante décadas la extracción de productos del bosque tanto maderables como no maderables ha sustentado y mantenido la economía de muchas comunidades humanas de México. En la Reserva de la biosfera "El Cielo" (RBEC), Gómez Farías, Tamaulipas, el cultivo agroforestal in-situ de la palma "camedor" (Chamaedorea radicalis Mart.) inició como una alternativa socio-económica en los años 90, con ventajas ambientales al detener la deforestación (desde los años 40 solo se recolectaba), ya que para su crecimiento requiere de la sombra de acahuales y bosques (Quero 1994). La siembra se realiza en dos temporadas, una en el mes de marzo, emergiendo las plántulas para el mes de junio y otra en el mes de julio, apareciendo los primeros brotes en octubre (Trejo 2003). Un estudio previo sobre la herbivoría en $C$. radicalis, dentro de dos tipos de
Lara-Villalón, M., Rosas-Mejía, M., Rojas-Fernández, P. \& ReyesCastillo, P. 2015. Ants (Hymenoptera: Formicidae) associated with the camedor palm (Chamedorea radicalis Mart.) in the tropical forest of Gómez Farías, Tamaulipas, Mexico. Acta Zoológica Mexicana (n. s.), 31(2): 270-274.

ABSTRACT. Ant's diversity associated to an agroforestry Chamaedora radicalis Mart., palm culture has not been studied in El Cielo Biosphere Reserve's Tropical Forest. Ants were collected from the palm fronds, using an entomological net the following species during two seasons: Odontomachus laticeps, Crematogaster cerasi, Camponotus atriceps, Temnothorax texanus, Tapinoma sp., y Pheidole arctos, weth season. Wasmannia auropuntata, Camponotus atriceps, Monomorium floricola, Pheidole arctos y Solenopsis geminate, dry season. Species previously recorded by other authors for RBEC are compared. Overall genera found are agro ecosystem colonizers, their food habits are foraging, hunters, nectar collectors, seed, and dead animal debris. Organism knowledge associated to camedor palm and its environment will allow establishing an integrated pest management for this important bioresource sustainability.

Key words: diversity, species records, pests.

vegetación en la RBEC (bosque tropical subcaducifolio y bosque mixto templado) sugiere que independientemente del tipo de vegetación $C$. radicalis constituye una parte importante del sotobosque, esto es 1900 plantas/ha para el bosque tropical subcaducifolio y 1760 plantas/ha para el bosque mixto templado. En cuanto al daño foliar, el mayor registro visual fue atribuible al grupo de los insectos herbívoros (Sánchez-Ramos et al. 2010). Este hallazgo concuerda con Oyama \& Dirzo (1991), donde los autores identificaron ocho especies de Calyptocephala (Coleoptera-Chrysomelidae) como los principales herbívoros asociados latitudinalmente a la distribución geográfica de las especies de Chamaedorea a través de un gradiente desde Brasil hasta México. Otros crisomélidos especialistas como Epitrix sp. dañan el follaje ocasionando perforaciones, defoliación de bordes, puntas y raspado del 
haz (Cisneros 1992). En la RBEC, el primer estudio sobre los insectos asociados al cultivo agroforestal de palma camedor que causan daño económico es el de Lara et al. (2012) quienes encontraron varios órdenes y familias de insectos. Del grupo de las hormigas, Ruiz (1993), reporta a la "hormiga arriera" Atta mexicana (F. Smith), como importante defoliadora de árboles frutales. López (1993) observó que Solenopsis sp. daña el follaje en las huertas de durazno del ejido “Alta Cima”. Justino y Phillips (1992) compararon la riqueza de especies de formícidos entre el Bosque subtropical y los cultivos de mango, cítricos y nopal, reportando por primera vez para esta reserva, la presencia de la hormiga invasora Wasmannia auropunctata, además de otras 26 especies. También la mirmecofauna asociada a huertas de mango fue estudiada por FloresMaldonado y González-Hernández (2005), encontrando 27 especies. Aparte de lo registrado en estos estudios, no existe información sobre las hormigas de otros ambientes dentro de la RBEC, tales como los bosques de pino y de pino-encino, los matorrales, la vegetación de galería, o los pastizales; ni tampoco de otros cultivos.

En los últimos años, la demanda de hojas de palma camedor en el mercado nacional e internacional ha aumentado en más del $100 \%$, por lo cual el gobierno estatal de Tamaulipas ha promovido en la RBEC la instalación in-situ de cultivos agroforestales certificados. Para el adecuado manejo de estos cultivos es necesario conocer a su entomofauna asociada, principalmente a sus potenciales defoliadores y a las especies útiles en el Manejo Integrado de Plagas.

El presente constituye un reporte del estudio de las hormigas (Formicidae) asociadas al cultivo agroforestal de la palma camedor Chamaedorea radicalis (Mart.), en el bosque tropical de la Reserva de la Biosfera El Cielo (RBEC).

\section{MATERIALES Y MÉTODOS}

El estudio se hizo en un cultivo de palma camedor en el

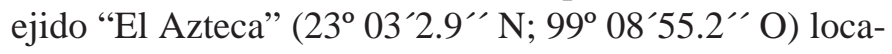
lizado dentro de la RBEC. El cultivo se encuentra bajo el dosel arbóreo compuesto principalmente por elementos del bosque tropical utilizados como sombra o nodriza, como son: Guazuma ulmifolia Lam., Cedrela odorata L., Lysiloma microphyllum Benth, Bursera simaruba (L.) Sarg., Enterolobium cyclocarpum Griseb., Brosimum alicastrum Sw. (Mora-Olivo et al. 1997) (Figura 1). La temperatura media anual para este bosque varía entre $39^{\circ} \mathrm{C}$ y $4.4^{\circ} \mathrm{C}$; época lluviosa con precipitaciones máximas de $2856 \mathrm{~mm}$ (abril-octubre) época seca con precipitaciones mínimas de 1175 mm (nov-marzo).
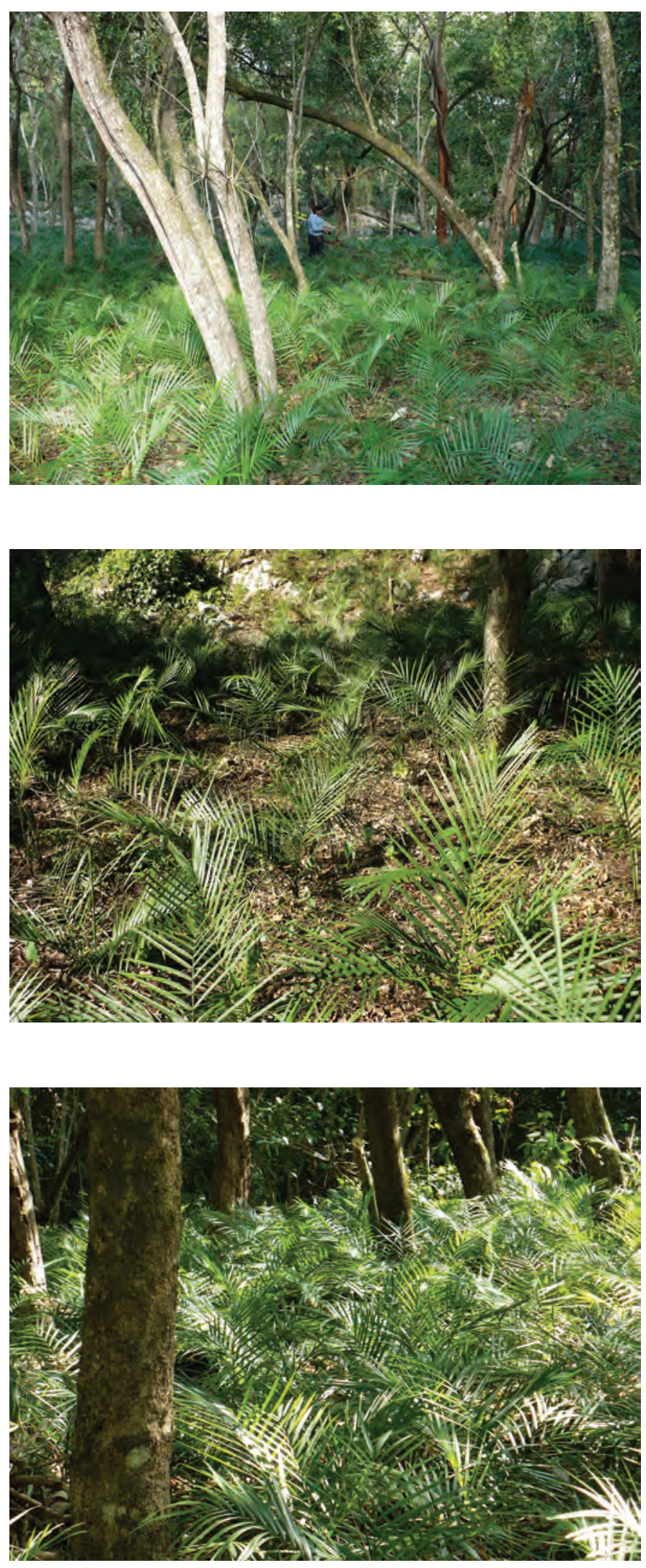

Figura 1. Cultivo de palmilla Chamaedorea radicalis en sistema agroforestal en la Reserva de la Biosfera El Cielo, Tamaulipas, México. 
Se delimitaron cuatro parcelas de $50 \times 100$ m (5000 $\mathrm{m}^{2)} \mathrm{y}$ en cada una de ellas se realizó un muestreo con red entomológica de golpeo, realizando 100 golpes desplazándose en forma de zigzag sobre el área de la parcela, para cubrir el máximo de follaje a muestrear. Los muestreos se realizaron (8/06/2012) en la y otra en (20/12/2012). Cada muestra fue fijada con alcohol etílico al 70\%. Las hormigas se montaron en seco y se identificaron utilizando bibliografía especializada (Baroni-Urbani 1978; Brown 1978; Buren 1968; Fernández 2002; Longino y Fernández 2008; Smith 1965; Trager 1991; Wilson 2003) y un microscopio estereoscópico Carl Zeiss (Stemi SV6).

\section{RESULTADOS Y DISCUSIONES}

En general las especies de hormigas encontradas, no representan un problema fitosanitario con pérdida económica para el cultivo de la palma camedor $C$. radicalis, ya que sus hábitos de alimentación no impactan las frondes de la planta ni sus frutos.

Se colectaron en total nueve especies de nueve géneros y tres subfamilias, de las cuales se encontraron en época lluviosa a Odontomachus laticeps, Crematogaster cerasi, Camponotus atriceps, Temnothorax texanus, Tapinoma sp. y Pheidole arctos. En época seca a Wasmannia auropunctata, Camponotus atriceps, Monomorium floricola, Pheidole arctos y Solenopsis geminata. En ambas épocas se presentaron $C$. atriceps y $P$. arctos.

A continuación se describen las especies encontradas y se comentan aspectos sobre su biología y ecología.

\section{Subfamilia Formicinae}

Camponotus Mayr, 1861. Hormigas de tamaño pequeño a muy grandes. El margen posterior del clípeo está alejado de los alvéolos antenales por una distancia igual o mayor al diámetro de estos alvéolos. No hay abertura de la glándula metapleural. Mesosoma convexo, variable entre las especies. Forrajeros generalistas. Género cosmopolita (Brown, 2000; Fernández, 2003a).

Camponotus atriceps (Smith, 1858). Especie común y de amplia distribución en las zonas tropicales y subtropicales de América (conocida en la literatura por su sinónimo C. abdominalis). Es generalista, tanto en su alimentación como en sus sitios de anidación, y puede vivir en sitios con elevado disturbio, incluyendo ambientes urbanos y domésticos. En México se distribuye en todo el país desde el nivel del mar hasta los 2000 m (Rojas-Fernández 2011). En Veracruz se le considera plaga doméstica y hay registros de que en Brasil ataca apiarios (Campos-Farinha 2005).

\section{Subfamilia Myrmicinae}

Monomorium Mayr, 1855. Pequeñas a moderadas en tamaño, antenas con 11 artejos y maza antenal compuesta por tres, clípeo bicarinado. Los individuos de este género en Norteamérica son por lo general de color negro y brillante. Son generalistas, especialistas de climas cálidos, fríos y tropicales. (Brown 2000; Fisher \& Cover 2007).

Monomorium floricola (Jerdon, 1851). Especie originaria del Viejo Mundo introducida a los trópicos americanos. Especie que es muy común en vegetación secundaria y sitios perturbados, incluyendo habitaciones humanas.

Pheidole Westwood, 1839. Uno de los géneros más comunes, antenas compuestas de 12 artejos con una maza diferenciada de tres artejos. Mandíbulas con numerosos dientes y dentículos. Obreras normalmente dimórficas, a veces polimórficas, los soldados presentan cabeza grande, que a veces sobrepasa el tamaño del cuerpo. Formícido generalista. Cosmopolita (Brown 2000; Fernández 2003b; Fisher \& Cover 2007).

Pheidole arctos Wilson, 2003. Componente de la fauna Neártica, esta especie se le conoce solamente de su localidad tipo (MEXICO: Gómez Farías, Tamaulipas, 400$600 \mathrm{~m}$ ). No se conoce nada acerca de su biología (Wilson 2003).

Solenopsis Westwood, 1840. Hormigas de tamaño pequeño, monomórficas o dimórficas. Se reconocen fácilmente por las antenas, compuestas por 10 artejos, con una maza de dos. Clípeo bicarinado. El propodeo carece de dientes o espinas. Especies crípticas, especialistas de climas tropicales. Forrajeras generalistas y depredadoras. Cosmopolitas (Fernández 2003b; Fisher \& Cover 2007).

Solenopsis geminata (Fabricius, 1804). De origen sudamericano, esta especie se dispersó de manera natural hacia el norte llegando a México y hasta el sureste de Estados Unidos (Trager 1991). Es una de las seis hormigas invasoras más importantes a nivel mundial (McGlynn 1999). Anida en el suelo en sitios abiertos de ecosistemas perturbados, principalmente en cultivos y potreros, en donde frecuentemente es la especie dominante (Carroll \& Risch 1983, Perfecto 1991). Se han documentado sus efectos nocivos y benéficos en los cultivos agrícolas pero se sabe muy poco de sus poblaciones en los bosques naturales o en plantaciones agroforestales.

Temnothorax Mayr, 1861. Hormigas con cinco dientes en las mandíbulas y con 11 o 12 artejos antenales. Las hormigas de este género formaban parte de Leptothorax. (Brown 2000; Fisher \& Cover 2007).

Temnothorax texanus (Wheeler, 1903). Esta es especie de la fauna Neártica. Es común y vive en sitios con poca cobertura vegetal. No se sabe nada acerca de su biología, pero puede especularse con base en la biología de otras especies afines, que se alimenta de exudados azucarados 
producidos por plantas e insectos hemípteros o de insectos muertos (Mackay 2000).

Wasmannia Forel, 1893. Hormigas pequeñas. Parte frontal de la cabeza con rúgulas irregulares longitudinales. Porciones laterales del clípeo forman una carina bien definida en frente de las inserciones antenales. Especialistas de climas tropicales. Forrajeras generalistas, habitantes de la hojarasca, anidando en suelo, árboles o troncos en descomposición. (Brown 2000; Fernández 2003b).

Wasmannia auropunctata (Roger 1863). Esta especie es nativa de las selvas del Neotrópico y se distribuye desde el noreste de México hasta Sudamérica (Wetterer \& Porter 2003). Introducida por el hombre a los trópicos del resto del mundo, en muchos lugares causa serios daños a la fauna nativa, incluyendo a las hormigas (Clark et al. 1982, Jourdan 1997). Aunque es común en los bosques naturales, es en los sitios perturbados y los cultivos agrícolas en donde se vuelve muy abundante y llega a convertirse en plaga al favorecer las poblaciones de hemípteros (de Souza et al. 1998).

\section{Subfamilia Ponerinae}

Odontomachus Latreille, 1804. Mandíbulas alargadas con dientecillos en el ápice, y carina nucal formando una $\mathrm{V}$, con una línea cefálica longitudinal media. El nodo del peciolo puede ser cónico y puntiagudo apicalmente. Depredadoras especializadas. Oportunistas. (Brown 2000; Fisher \& Cover 2007; Lattke 2003; Mackay \& Mackay 1989).

Odontomachus laticeps (Roger, 1861). Especie centroamericana propia de selvas tropicales y subtropicales. Se distribuye desde el noreste de México hasta Panamá (Kempf 1972, Brandao 1991). Es depredadora y caza individualmente en la hojarasca y en la vegetación baja.

Dentro de las especies que se colectaron en época lluviosa, Odontomachus y Pheidole se han registrado (Escobar et al. 2007) como dispersoras de semillas y para época de seca Pheidole cumple esta misma función ecológica. Como depredadoras de plagas potenciales se han documentado a Solenopsis, Crematogaster, Pheidole y Wasmannia (Way \& Khoo 1992, Varón et al. 2004). Aunque muchas especies de hormigas son depredadoras generalistas que al atacar plagas podrían ser valiosos agentes de control biológico. Wetterer (2010) y Dubois (1986) mencionan que Monomorium floricola, Solenopsis geminata y Tapinoma minutum, son enlistadas como depredadores de huevos de Lepidópteros nativos, causando reducción de las poblaciones. Mera y colaboradores (2010) mencionan a especies de Temnothorax como potenciales depredadores de Cicadellidae y Chrysomelidae. También señalan asociaciones mutualistas de Camponotus con homópteros Pseudococcidae.
Las hormigas cumplen una gran variedad de funciones ecológicas en los ecosistemas debido a que por su abundancia y diversidad utilizan numerosos estratos de nidificación, tienen un amplio espectro de alimentación, son persistentes a pesar de las fluctuaciones temporales en la disponibilidad del alimento, se asocian con diferentes especies de plantas y animales además de tener influencia sobre la formación del suelo y las relaciones suelo-planta (Davidson \& Mckey 1993, Rojas-Fernández 1994, Risch \& Carrol 1982) y en los agroecosistemas estimulan la presencia de especies benéficas de control biológico (Way \& Khoo 1992).El conocimiento de los organismos asociados a la palmilla o palma camedor en su ambiente dentro de la Reserva de la Biosfera El Cielo, permitirá establecer un manejo integrado de plagas que asegure la sustentabilidad de este importante bio-recurso.

AGRADECIMIENTOS. Al proyecto Capacitación en técnicas de cultivo para palma camedor (Chamaedorea radicalis Mart.) como estrategia para la conservación y aprovechamiento sustentable en la Reserva de la biosfera El Cielo, auspiciado por: World Wildlife FoundUAT-Asociación de palmilleros del Sur de Tamaulipas, 2011-2012.

\section{LITERATURA CITADA}

Baroni Urbani, C. 1978. Materiali per una revisione dei Leptothorax neotropicali apparenti al sottogenere Macromischa Roger, n. comb. (Hymenoptera: Formicidae). Entomologica Basiliensis, 3: 395-618.

Brown, W. L. 2000. Diversity of Ants, pp. 45-79. In: Agosti, D., J. D. Majer, L. E. Alonso \& T. R. Schultz (Eds.). Ants: standard methods for measuring \& monitoring biodiversity. Smithsonian Institution Press, Washington.

Brown, W. L., Jr. 1978. A supplement to the world revision of Odontomachus (Hymenoptera, Formicidae). Psyche, 84: 281-285.

Buren, W. F. 1968. A review of the species of Crematogaster, sensu stricto, in North America (Hymenoptera, Formicidae). Part II. Descriptions of new species. Journal of the Georgia Entomological Society, 3: 91-121.

Campos-Farinha, A. E. 2005. Urban pest ants of Brazil (Hymenoptera: Formicidae), pp. 81-84. In: Lee, C.Y. \& Robinson, W.H. (Eds.). Proceedings of the 5th International Conference on Urban Pests, Suntec, Singapore, 10-13 July 2005. Perniagaan Ph’ng, Malaysia.

Carroll, C. R. \& Risch, S. J. 1983: Tropical annual cropping systems: ant ecology. Environmental Management, 7: 51-57.

Castro, A. G., Queiroz, M. V. B. \& Araújo, L. M.1990. O papel do distúrbio na estructura de comunidades de formigas (Hymenoptera: Formicidae). Revista Brasileira de Entomologia, 34: 201-213.

Cisneros, F. H. 1992. El manejo integrado de plagas. Guía de Investigación. CIP 7. Centro Internacional de la Papa, Lima, Perú. 38 pp.

Clark, D. B., Guayasamin, C., Pazamino, O., Donoso, C. \& Paez De Villacis, Y. 1982. The tramp ant Wasmannia auropunctata: Autecology and effects on ant diversity and distribution on Santa Cruz Island, Galapagos. Biotropica, 14: 196-207.

Davidson, D. \& McKey, D. 1993. The evolutionary ecology of symbiotic ant-plant relationships. Journal of Hymenoptera Research, 2: 18-33. 
De Souza, A. L. B., Delabie, J. H. C. \& Fowler, H. G. 1998. Wasmannia spp. (Hym. Formicidae) and insect damages to cocoa in Brazilian farms. Journal of Applied Entomology, 122: 339-341.

Dubois, M. B. 1986. A revision of the native New World species of the ant genus Monomorium (minimum group) (Hymenoptera: Formicidae). University of Kansas Science Bulletin, 53: 65-119.

Escobar, S., Armbrecht, I. \& Calle, Z. 2007. Transporte de semillas por hormigas en bosques y agroecosistemas ganaderos de los Andes Colombianos. Agroecología, 2: 65-74.

Fernández, F. 2002. Revisión de las hormigas Camponotus del subgénero Dendromyrmex (Hymenoptera:Formicidae). Papéis Avulsos de Zoologia (São Paulo), 42: 47-101.

Fernández, F. 2003a. Subfamilia Formicinae, pp. 299-306. In: Fernández, F. (Ed.). Introducción a las hormigas de la región Neotropical. Instituto de Investigación de Recursos Biológicos Alexander von Humboldt, Bogotá, Colombia.

Fernández, F. 2003b. Subfamilia Myrmicinae, pp. 307-330. In: Fernández, F. (Ed.). Introducción a las hormigas de la región Neotropical. Instituto de Investigación de Recursos Biológicos Alexander von Humboldt, Bogotá, Colombia.

Fisher, B. L. \& Cover, S. P. 2007. Ants of North America: A guide to the genera. Berkeley \& Los Angeles, California.

Flores-Maldonado, K. \& González-Hernández, H. 2005. La mirmecofauna en arboles de mango, pp. 483-488. In: Sanchez-Ramos, G., P. Reyes-Castillo y R. Dirzo 2005. (Eds.) Historia Natural de la Reserva de la Biosfera El Cielo.

Jourdan, H. 1997. Threats on Pacific islands: the spread of the tramp ant Wasmannia auropunctata (Hymenoptera: Formicidae). Pacific Conservation Biology, 3: 61-64.

Jusino-Atresino, R. \& Phillips Jr., S.A. 1992. Myrmecofauna en la reserva ecológica de la biosfera "El Cielo", Tamaulipas, Mexico. Biotam, 4: 41:54.

Lara-Villalón, M., Sánchez-Ramos, G., Gaona-García, G. \& Trejo-Hernández, L. 2012. Plagas potenciales para el cultivo de la palma camedor Chamaedorea radicalis Mart. en la reserva de la biosfera el cielo. Tríptico informativo para productores de palmilla. 1 pag. World Wildlife Found-UAT-Asociación de palmilleros del sur de Tamaulipas.

Lattke, J. E. 2003. Subfamilia Ponerinae, pp. 261-276. In: F. Fernández (Ed.). Introducción a las hormigas de la región Neotropical. Instituto de Investigación de Recursos Biológicos Alexander von Humboldt, Bogotá, Colombia.

Longino, J. T.\& Fernández, F. 2007. Taxonomic review of the genus Wasmannia. (pp. 271-289). In: Snelling, R. R., B. L. Fisher, y P. S. Ward (Eds). Advances in ant systematics (Hymenoptera: Formicidae): homage to E. O. Wilson- 50 years of contributions. Memoirs of the American Entomological Institute.

López de León, R. 1993. La fruticultura en la zona de amortiguamiento e influencia de la reserva de la biosfera el cielo. In: Memorias. Segundo Curso Internacional sobre Entomología Tropical en la Reserva de la Biosfera El Cielo. UAT., pp. 51-55.

Mackay, W. P. \& Mackay, E. 1989. Clave para los géneros de hormigas en México (Hymenoptera: Formicidae). pp. 1-82. In: Quiroz L. N. \& L. M. P. Garduño (Eds.). Memorias II Simposio Nacional de Insectos Sociales. Oaxtepec, Morelos.

MacKay, W. P. 2000. A review of the New World ants of the subge- nus Myrafant, (genus Leptothorax) (Hymenoptera: Formicidae). Sociobiology, 36: 265-444.

McGlynn, T. P. 1999: The worldwide transfer of ants: geographical distribution and ecological invasions. Journal of Biogeography, 26:535-548.

Mera, V. Y. A., Gallego, R. G. M. C. \& Armbrecht, I. 2010. Interacciones entre hormigas e insectos en follaje de cafetales de sol y sombra, cauca Colombia. Revista Colombiana de Entomologia, 36: 116-126.

Mora-Olivo, A., Mora-López, J. L., Jiménez, J. \& Sifuentes, J. 1997. Vegetación y flora asociada a la palmilla (Chamaedorea radicalis Mart.) en la Reserva de la Biosfera El Cielo. Biotam, 8: $1-10$.

Oyama, K. \& Dirzo, R. 1991. Ecological aspects on the interaction between Chamaedorea tepejilote, a dioecious palm and Calyptocephala marginipennis, an herbivorous beetle, in a Mexican rain forest. Principes, 35: 86-93.

Perfecto, I. 1991: Dynamics of Solenopsis geminata in a tropical fallow field after ploughing. Oikos, 62:139-144.

Quero, H. J. 1994. Flora de Veracruz Fascículo No 81. Palmae. Instituto de Ecología, A.C., Xalapa, Veracruz, México. 118 pp.

Risch, S. J. \& Carroll, C. R. 1982. Effect of a keystone predaceous ant, Solenopsis geminata, on arthropods in a tropical agroecosystem. Ecology, 63: 1979-1983.

Rojas-Fernández, P. 2001. Las hormigas del suelo en México: diversidad, distribución e importancia (Hymenoptera: Formicidae). Acta Zoológica Mexicana (n. s.), 1: 189-238.

Ruiz, C. E. 1993. Plagas de cultivos tropicales en la zona de la reserva El Cielo y el control biológico, pp. 6-12.. In: Memorias. Segundo Curso Internacional sobre Entomología Tropical en la Reserva de la Biosfera El Cielo. UAT.

Sánchez-Ramos, G., Reyes-Castillo, P., Mora Olivo, A. \& Martínez-Ávalos, J. G. 2010. Estudio de la herbivoría de la palma camedor (Chamaedorea radicalis) Mart., en la Sierra Madre Oriental de Tamaulipas, México. Acta Zoológica Mexicana (n. s.), 26: 153-172.

Smith, M. R. 1965. House-infesting ants of the eastern United States, their recognition, biology and ecology importance. United States Department of Agriculture,Technical Bulletin, 1326: 1-105 pp.

Trager, J. C.1991. A revision of the fire ants, Solenopsis geminata group (Hymenoptera: Formicidae: Myrmicinae). Journal of the New York Entomological Society, 99: 141-198.

Trejo, 2003. Memoria de Resúmenes del VII Simposio Internacional y II Congreso Nacional de Agricultura Sostenible. “Análisis de la sostenibilidad en la agricultura”, pp. 126.

Varón, H. E., Hanson, P., Borbón, O., Carballo, M. \& Hilje, L. 2004. Potencial de Hormigas como depredadores de la broca del Café (Hypothenemus hanpei) en Costa Rica. Manejo Integrado de Plagas y Agroecología (Costa Rica), No. 73: 42-50.

Way, M. J. \& Khoo, K. C. 1992. Role of ants in pest management. Annual Review of Entomology, 37: 479-503.

Wetterer, J. K.2010. Worlwide spread of the flower ant. Monomorium floricola (Hymenopter: Formicidae). Mirmecological news, 19-27.

Wilson, E. O. 2003. Pheidole in the New World: a dominant, hyperdiverse ant genus. Cambridge, MA: Harvard University Press. 